\title{
Particles Size Estimation of Polydopamine Based Polymeric Nanoparticles Using Near-Infrared Spectroscopy Combined with Linear Regression Method
}

\author{
Nahla Rahoui, Mohammad Hegazy, Bo Jiang, Nadia Taloub, Yu Dong Huang \\ MIIT Key Laboratory of Critical Materials Technology for New Energy Conversion and Storage, School of Chemistry and \\ Chemical Engineering, Harbin Institute of Technology, Harbin, China \\ Email: nahlarahoui@hit.edu.cn, jiangbo5981@hit.edu.cn, huangyd@hit.edu.cn
}

How to cite this paper: Rahoui, N., Hegazy, M., Jiang, B., Taloub, N. and Huang, Y.D. (2018) Particles Size Estimation of Polydopamine Based Polymeric Nanoparticles Using Near-Infrared Spectroscopy Combined with Linear Regression Method. American Journal of Analytical Chemistry, 9, 273-285.

https://doi.org/10.4236/ajac.2018.95021

Received: April 18, 2018

Accepted: May 21, 2018

Published: May 24, 2018

Copyright ( $\odot 2018$ by authors and Scientific Research Publishing Inc. This work is licensed under the Creative Commons Attribution International License (CC BY 4.0).

http://creativecommons.org/licenses/by/4.0/ (c) (i) Open Access

\begin{abstract}
The particle size is considered as a fundamental property of nanoparticles used for cargo delivery (CDS) purpose. The objective of this study was the particles size quantification of polydopamine nanoparticles (PDANS) via the near infrared spectroscopy (NIRS) combined with chemometrics tools. The successful synthesis of PDANS was proved using several characterization methods. Compared with the model extracted using raw spectral data, the accuracy and stability of the new model extracted from pre-processed data were significantly improved. The PDANS particle size samples have been predicted with acceptable accuracy. The correlation $\left(R^{2}\right)$ between NIRS and the TEM granulometrie data was $99.81 \%$, while the root mean square error of calibration RMSEC was $0.196 \mathrm{~nm}$. This research shows that NIRS combined with a regression method is a viable tool for the quality determination of $C D$ system.
\end{abstract}

\section{Keywords}

Near Infrared Spectroscopy, Particle Size Effect, Nanoparticles, Linear Regression

\section{Introduction}

IR spectroscopy plays an important role for evaluation of drug quality in industrial and research process especially for organic ingredient with abundant $\mathrm{CH}$, $\mathrm{OH}, \mathrm{SH}$, or NH groups. Near-Infrared Spectroscopy (NIRS) $(780-2595 \mathrm{~nm})$, is a noninvasive and eco-friendly analytical technique that presents a promising alternative for cargoes delivery system (DDS) qualitative and quantitative analysis [1] [2]. NIRS is known as a rapid and nondestructive analytical method with 
no sample preparation [2]. The sensitivity of NIRS spectral signals to any physical factors including particle size, the crystallinity ratio, solid surface roughness, coating thickness, temperature [1] [3] [4] [5] [6] [7] and chemical parameters such as analytes ratio, moisture ratio [8], concentrations and chemical composition; the ingredient distribution [9] could be of great interest in order to understand and evaluate new designed DDS.

For any drug delivery system, the determination of drug and the container physiochemical properties is vital for estimation of in vivo delivery and the study of the kinetics and release behaviour [10]. A good characterization of the deigned system could lead to a good understanding to the designed system, by the way an accurate estimation of the therapeutic effect [11]. Importantly, the drug substrate design and a precise investigation of the physiochemical properties including particle size are primordial for understanding of the possibility of DDS leakage by enhanced Plasmon Resonance effect (EPR) [12] [13], besides it capacity of drug loading in case of chemotherapy or photothermal or photodynamic effect in case of other physical therapeutic modalities [10].

The determination of particle characteristics using conventional electronic microscope of laser diffractometer with high accuracy and reproducibility was always challenging inversion problem [14], besides the nonavailability of such apparatus in every lab. NIRS instead is largely available apparatus and noninvasive quantitative and qualitative analysis method. This reliable and precise estimation of the DDS physiochemical properties could be achieved by NIRS as a nondestructive method and a precise quantitative tool when chemometrics is used.

The paper is structured around two main points presented two parts one art is the characterization results of the designed PDA based nanoparticles (PDANS), prepared for further drug delivery purposes. The accuracy of the nanostructures size and morphology is primordial for biomedical application [12], thus a set of prepared PDANS of multiple particles size was prepared and analysed for computational modelling using near infrared spectroscopy. The NIRS spectra of polymeric nanoparticles is considered as a response of the inherent absorption properties caused by its chemical component such chemical structure or analyte ratio and scattering phenomena related to its physical characteristics such as particles' size and morphology [1] [2]. The NIRS spectral data are complex and need further chemometrics methods for extraction and interpretation of the desired information [3] [9].

In the present study, in order to prove the importance of NIRS for various DDS properties estimation, partial least square method (PLS)were used to evaluate the NIR spectra acquired for PDANS particle size and correlate it to transmission electron microscope primary analysis method. The accuracy of modelling process was by several correlation indexes.

\section{Materials and Methods}

\subsection{Reactants}

Dopamine hydrochloride DA-HCl ( 35\% - 37\%) was purchased from Aladdin. 
Sodium hydroxide $(\mathrm{NaOH})(\geq 96 \%)$ were obtained from Sinopharm Chemical Reagent. The ultrapure water used for solution preparation was prepared using Millipak filter of $0.22 \mu \mathrm{m}$ pore size Millipore in Milli-Q system. Ethanol absolute was purchased from Tianjin Hengxing Chemical preparation. All chemical reagents were used as received without any purification.

\subsection{Methods}

\subsubsection{Preparation of Polydopamine Nanoparticles}

The PDA nanoparticles (PDANS) with different particles size were prepared by the oxidation and self-polymerization of dopamine hydrochloride [15]. Briefly, different amount $(\mathrm{mg})$ of dopamine hydrochloride was dissolved in specific volume " $\mathrm{VdiH}_{2} \mathrm{O}$ " ( $\mathrm{mL}$ ) of deionized water, at various temperatures and under vigorous stirring, a volume $\mathrm{VNaOH}$ of $1 \mathrm{~N} \mathrm{NaOH}$ solution was added. After $5 \mathrm{~h}$, the obtained dark black solution was centrifuged $(13,000 \mathrm{rpm})$ and then washed several times with deionized water. All large-size materials were removed by $4000 \mathrm{rpm}$ centrifugation. The obtained samples were washed several time with deionized water and ethanol, and then freeze-dried to form black powder. The optimized experimental conditions can be scaled up to obtain different size of PDANS particles as summarized in Table 1.

\subsubsection{General Characterization Method}

Fourier transform infrared (FTIR) spectra were recorded on a Nicolet FT-IR 5700 spectrometer the samples were prepared using potassium bromide $(\mathrm{KBr})$ pastille. Few milligrams of PDANS powder were mixed with $100 \mathrm{mg}$ of $\mathrm{KBr}$. The

Table 1. Experimental condition for synthesis polydopamine nanoparticles.

\begin{tabular}{cccccc}
\hline \multicolumn{5}{c}{ Experimental Conditions } \\
\hline Sample N & DA-HCl mass $(\mathrm{mg})$ & $\mathrm{VdiH}_{2} \mathrm{O}(\mathrm{mL})$ & $\mathrm{VNaOH} / \mathrm{n}: \mathrm{n}(\mu \mathrm{l})$ & $\mathrm{Rtemp}\left({ }^{\circ} \mathrm{C}\right)$ & $\mathrm{dTEM}(\mathrm{nm})$ \\
\hline 1 & 180.00 & 90.00 & $760 / 1: 0.65$ & 40 & $\sim \mathbf{1 3 5}$ \\
2 & 360.00 & 90.00 & $760 / 1: 0.32$ & 50 & $\sim 950$ \\
3 & 180.00 & 90.00 & $760 / 1: 0.65$ & 20 & $\sim \mathbf{1 7 0}$ \\
4 & 153.18 & 79.59 & $400 / 1: 0.40$ & 40 & $\sim \mathbf{2 8 5}$ \\
5 & 360.00 & 90.00 & $760 / 1: 0.32$ & 70 & $\sim 1350$ \\
6 & 153.18 & 79.59 & $400 / 1: 0.40$ & 25 & $\sim \mathbf{1 8 0}$ \\
7 & 119.70 & 90.00 & $312 / 1: 0.40$ & 30 & $\sim \mathbf{2 9 0}$ \\
8 & 153.18 & 79.59 & $600 / 1: 0.60$ & 30 & $\sim \mathbf{2 7 5}$ \\
9 & 180.00 & 90.00 & $094 / 1: 0.80$ & 50 & $\sim \mathbf{2 2 1}$ \\
10 & 270.00 & 90.00 & $880 / 1: 0.50$ & 30 & $\sim 650$ \\
11 & 360.00 & 90.00 & $1880 / 1: 0.80$ & 50 & $\sim \mathbf{5 2 0}$ \\
12 & 180.00 & 90.00 & $760 / 1: 0.65$ & 30 & $\sim \mathbf{2 5 0}$ \\
\hline
\end{tabular}

V: Volume; Rtemp: Room temperature; DA-HCl: Dopamine hydrochloride; dTEM: TEM particles diameter. 
FTIR were recorded for $2 \mathrm{~cm}^{-1}$ resolution over the range of $600-4000 \mathrm{~cm}^{-1}$ through diffuse reflection contact angle after 64 scans. UV-Vis full range absorption spectra were collected on a (Lambda 750S) spectrophotometer at $480 \mathrm{~nm}$ excitation wavelength.

\subsubsection{Modelling Reference Method}

Transmission electron microscope (TEM) images were performed on Hitachi $\mathrm{H}-7650$ microscope operating at an accelerating voltage of $100 \mathrm{kV}$. The samples were prepared by adding one drop of nanoparticles solution $(0.1 \mathrm{mg} / \mathrm{mL})$ onto a 300 mesh carbon film coated copper grid. The specimens were then air dried for 15 minutes. TEM have been used as reference method for multivariate analysis.

\subsubsection{Modelling Secondary Method}

A set of 12 samples of different particles size PDNS (As illustrate in Table 1). Every sample was ultrasonically dispersed in high purity water at the same concentration $(2 \mathrm{mg} / \mathrm{mL})$ and then its spectral data was collected on MATRIX-F BRUKER NIRS apparatus equipped with solid probe (as illustrated in photographs of Figure 1) with an integrating sphere. The samples were positioned in glass tubes and the probe was positioned perpendicularly on the bottom of the sample holder. The spectra of each sample were collected in the reflectance mode. Each spectrum was an average of 32 scans in the range of $4000-11,000$ $\mathrm{cm}^{-1}$. The data treatment was proceeded on OPUS 5.0 software. Furthermore, all the experiment was conducted at a temperature of $25^{\circ} \mathrm{C}$ and at humidity of $60 \%$. The mean of the three spectra collected from one sample was used for subsequent analysis. The NIRS data was used as secondary method.

\section{Results and Discussion}

Before you begin to format your paper, first write and save the content as a 12 samples of the PDA based polymeric nanoparticles were synthesized by neutralization of dopamine hydrochloride with $\mathrm{NaOH}$ and spontaneous autoxidation in air, which leads to a gradual formation of dark brown solution.

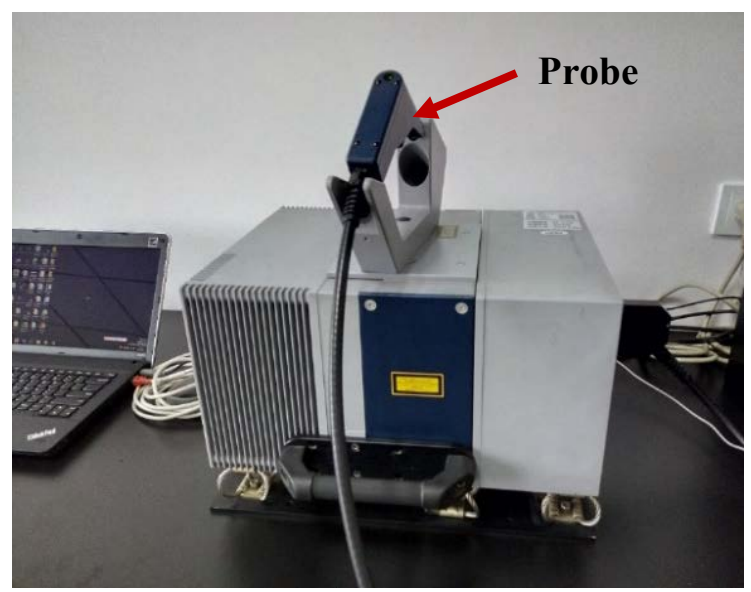

Figure 1. Photograph of NIRS equipment (with optic fibre probe). 
The prepared nanoparticles have been characterized and evaluated through various analysis method. The Figure 2 illustrates the experimental processed discussed in our manuscript.

\subsection{Structural Analysis of PDANS}

The PDANS FTIR spectra illustrated in Figure 3(a) reveals the presence of two peaks at $1621 \mathrm{~cm}^{-1}$ and $1500 \mathrm{~cm}^{-1}$ attributed to the characteristics absorption of the phenyl groups. The peaks at $1288 \mathrm{~cm}^{-1}$ and $1382 \mathrm{~cm}^{-1}$ are assigned to the scissoring of $\mathrm{CH}_{2}$ and $\mathrm{OH}$ of the PDA catechol groups, respectively [16] [17]. The wavelength band ranges from 2800 to $3700 \mathrm{~cm}^{-1}$ are assigned to the hydrox$\mathrm{yl}$ and amine group stretching of the dehydroxyindole units of PDA [2] [3]. The PDANS UV-visible spectrum shows a broad characteristic absorption peak at $208 \mathrm{~nm}$ as illustrated in Figure 3(b) [17].

\subsection{Morphological Analysis of Synthesised PDANS (Reference Method)}

Transmission electron microscope images of synthesised PDANSs as shown in Figure 3(c) and Figure 3(d) indicates well spherical and uniform shaped nanoparticles. The $135 \mathrm{~nm}$ sized particles presented in Figure 3(c) and Figure 3(d) have been used for FTIR and UV-Vis analysis. The average particle size of the all synthesised samples was organized in Table 1 . The results reveal that the particle diameter was highly affected by the concentration of dopamine hydrochloride (DA-HCl). The TEM particle size is directly proportional to the DA-HCl amount and inversely proportional to the reaction temperature and $\mathrm{NaOH}$ volume.

If the $\mathrm{NaOH}$ to $\mathrm{DA}-\mathrm{HCl}$ ratio is more then $1: 1$ the reaction product is just amorphous polydopamine and not nanoparticles. The TEM images for the all 12 samples are presented in Figure 4.

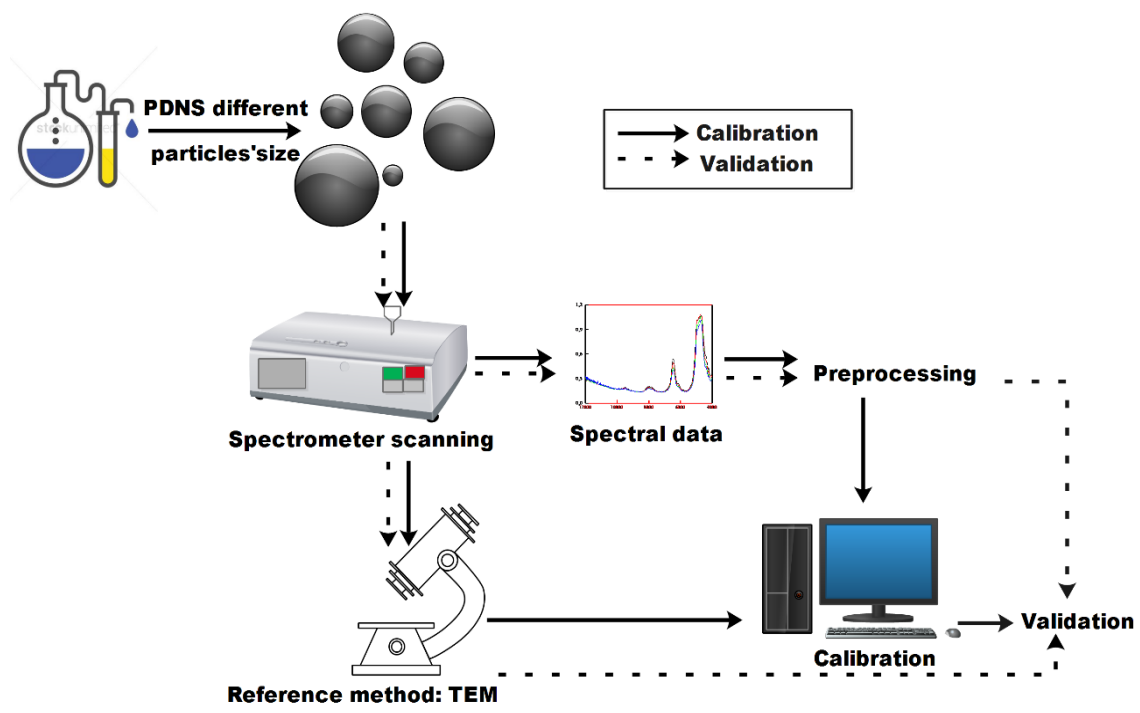

Figure 2. Schematic illustration of the experimental procedure. 


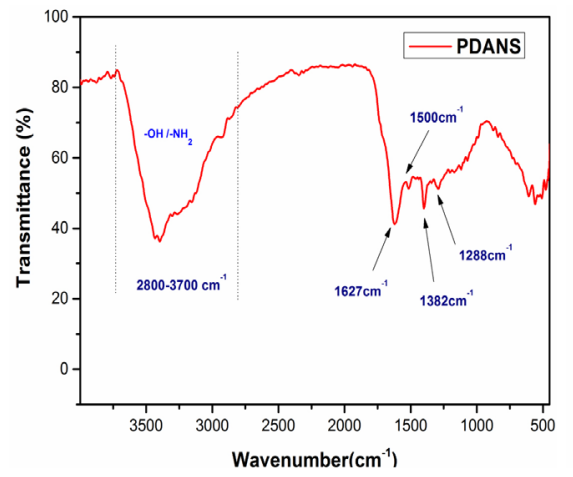

(a)

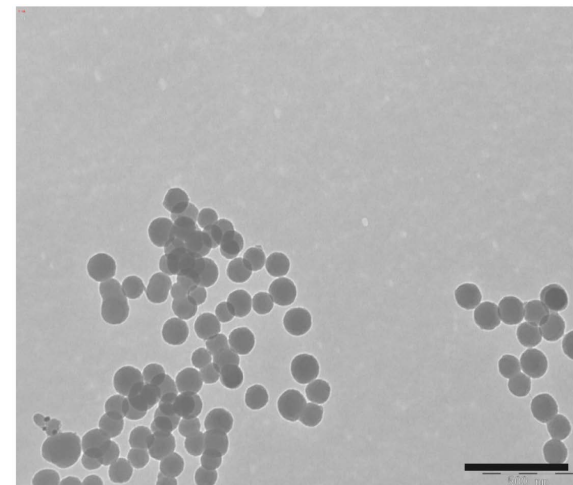

(c)

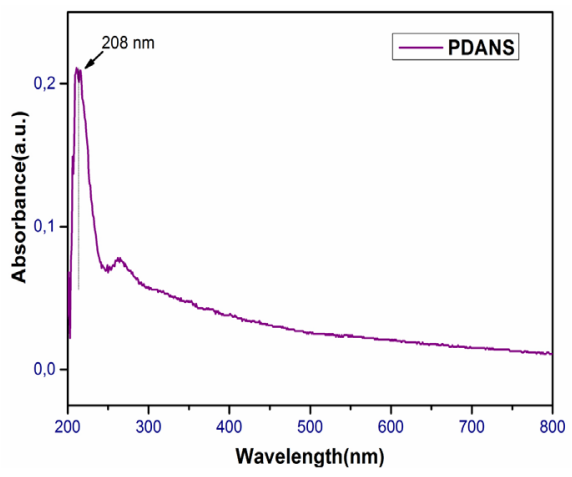

(b)

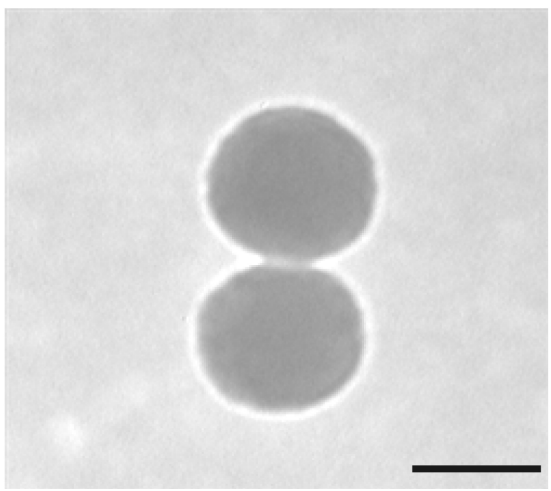

(d)

Figure 3. PDANS ( 135nm): (a) FTIR spectrum; (b) UV-Vis spectrum; (c) TEM micrograph, scale bare is $500 \mathrm{~nm}$; (d) TEM micrograph, scale bare is $100 \mathrm{~nm}$.
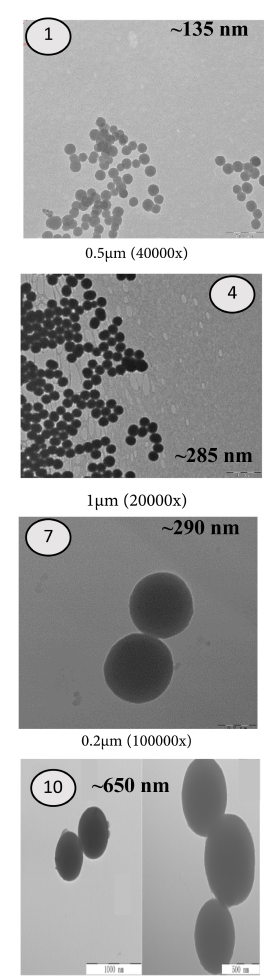

$0.5 \mu \mathrm{m}(40000 \mathrm{x}) / 0.5 \mathrm{um} 50000 \mathrm{x}$
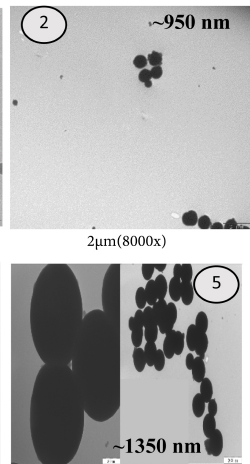

$0.5 \mu \mathrm{m}(40000 \mathrm{x}) / 2 \mu \mathrm{m}(10000 \mathrm{x})$

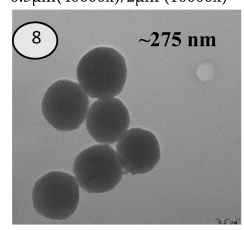

$0.2 \mu \mathrm{m}(70000 \mathrm{x})$

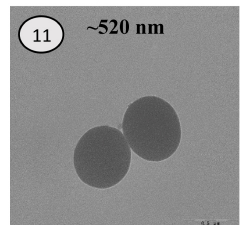

$0.5 \mu \mathrm{m}(60000 \mathrm{x})$

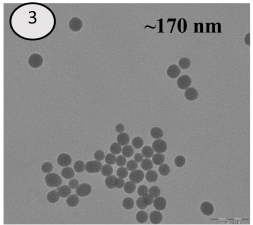

$0.5 \mu \mathrm{mn}(40000 \mathrm{x})$

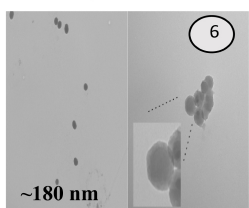

$1 \mu \mathrm{m}(20000 \mathrm{x}) / 0.2 \mu \mathrm{m}(100000 \mathrm{x})$

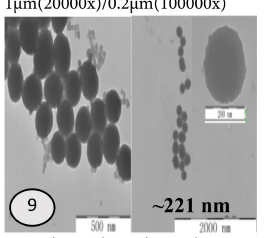

$500 \mathrm{~nm}-221 \mathrm{~nm}$

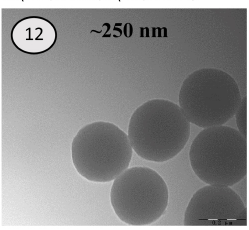

$0.2 \mu \mathrm{m}(120000 \mathrm{x})$

Figure 4. TEM images of synthesized PDANS at different particles size. 


\subsection{Computational Modelling of PDANS Particles' Size Using NIRS with Linear Regression Methods (Secondary Method)}

\subsubsection{Samples Preparation}

Previously synthesized samples as illustrated in Table 1 were collected and ultrasonically dispersed in high purity water at similar concentration of 2.5 $\mathrm{mg} / \mathrm{mL}$. The prepared samples were left in dark just before analysis at room temperature.

\subsubsection{Spectral Measurements}

About one drop of freshly prepared samples were scanned with NIRS using the optic fibre probe and saved in absorbance mode. A reference scan was taken once in every three samples. The NIR spectra were collected and saved as illustrated in Figure 5. The main features of the spectra are the absorption bands at [8925 - 7802], [7312 - 5986], [5380 - 4500] and $4029.7 \mathrm{~cm}^{-1}$, which are assigned to the second overtone of $\mathrm{C}-\mathrm{H}$ stretching of PDA catechol groups; first overtones of $\mathrm{O}-\mathrm{H}$ stretching of water and PDA phenyl groups and two combinations bands of $\mathrm{C}=\mathrm{O}$ stretching / second overtone of $\mathrm{O}-\mathrm{H}$ stretching and $\mathrm{CH} / \mathrm{NH}$ of quinone groups, respectively [10] [18]. The spectra reveal high similarities due the presence of the same chemical properties for the prepared PDANS. The slight variation in spectral data is mainly caused by the differences in the particles size.

\subsubsection{Spectra Preprocessing}

Before the start in the systematic procedure of calibration development, the raw spectra was treated using various preprocessing method including the baseline correction as illustrated in Figure 6(a) and the second derivative in order to achieve a better model performance. The standard normal variance is strongly not advised to use because it can eliminate the effect of the particle size effect on NIRS spectra [2], which could mistake our NIRS quantification data. The second derivative highest variations shown in Figure 6(b) are similar with the highest peaks in the raw NIR spectra around 5336, 5279, 5102, 4989 and $4095 \mathrm{~cm}^{-1}$.

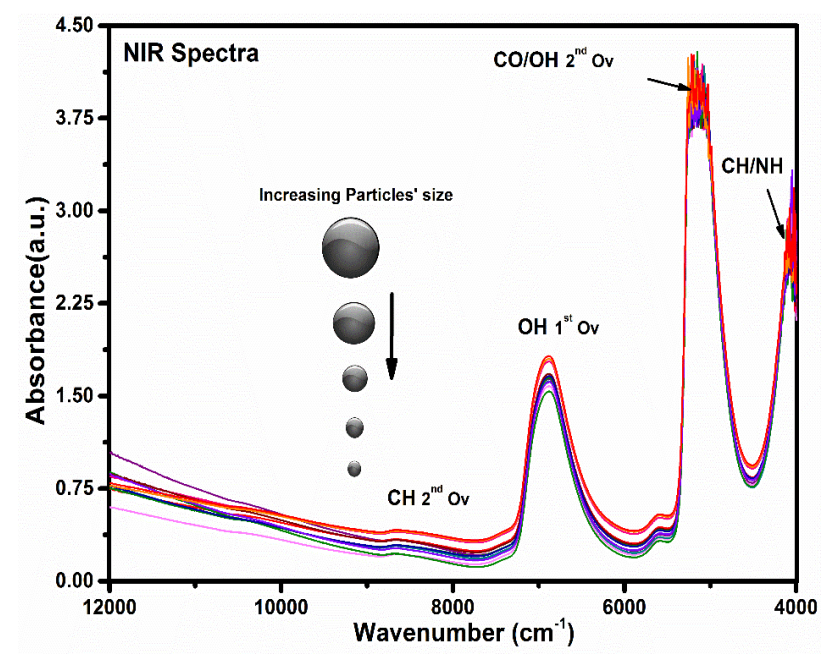

Figure 5. Raw NIR spectra of PDANS dispersion in high purity water of $2.5 \mathrm{mg} / \mathrm{mL}$. 


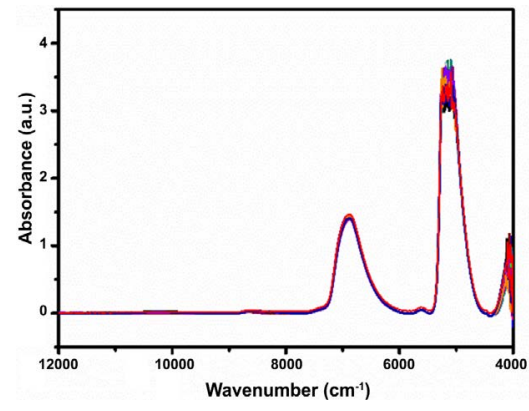

(a)

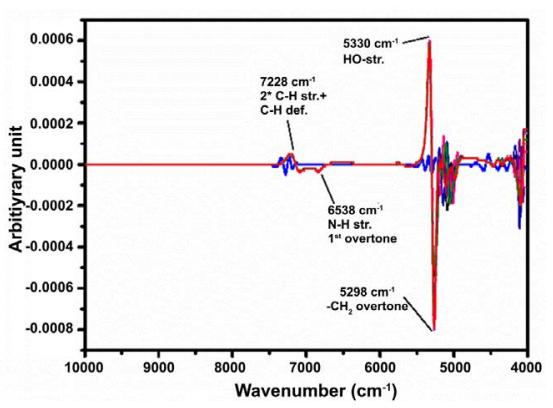

(b)

Figure 6. (a) NIR spectra after baseline correction using Rubberband Corrections; (b) second derivative NIR spectra of PDANS samples.

\subsubsection{The Selection of Calibration and Validation Set}

For the extraction of calibration and validation model, some statistical parameters should be calculated as index of the model performance, which are the model correlation coefficients $\left(R^{2}\right)$ and the root mean square error (RMSE). They were calculated using Equation (1), (2), (3) and (4) [19] [20]:

$$
R^{2}=\left\{1-\frac{\sum_{i=0}^{n}\left(y_{i}-\hat{y}_{i}\right)^{2}}{\sum_{i=0}^{n}\left(y_{i}-\bar{y}_{i}\right)^{2}}\right\}
$$

Calculated as the sum of squares about mean of $\mathrm{Y}$ over the sum of squares about regression line.

$$
R M S E C=\sqrt{\frac{\sum_{i=1}^{n}\left(\hat{y_{i}}-y\right)^{2}}{n-1}}
$$

Calculated as the square root of the sum of squares between true value (lab values) and the predicted values in case of cross validation.

$$
R M S E P=\sqrt{\frac{\sum_{i=1}^{m}\left(y_{i}-\hat{y}_{i}\right)^{2}}{m}}
$$

Calculated as the square root of the sum of squares between true value (lab values) and the predicted values by prediction model.

$$
R M S E E=\sqrt{\frac{1}{R-N-1} \sum_{i=1}^{n} \text { residues }_{i}^{2}}
$$

Calculated in case of test set validation determined from residues values. $R$ is also known as the number of PLS factors.

The determination of the optimum rank is primordial step before the extraction of the calibration model, it can be determined by plotting the $R^{2}, R M S E E$ and RMSECV values versus the rank. The determination of optimal rank simplified the model development process and performs the model selection.

The determination of the optimized factor of the model variable matrix was analysed and presented in the Figure 7(a) and Figure 7(b) for the calibration and validation models, respectively. The results reveals an optimized factor 


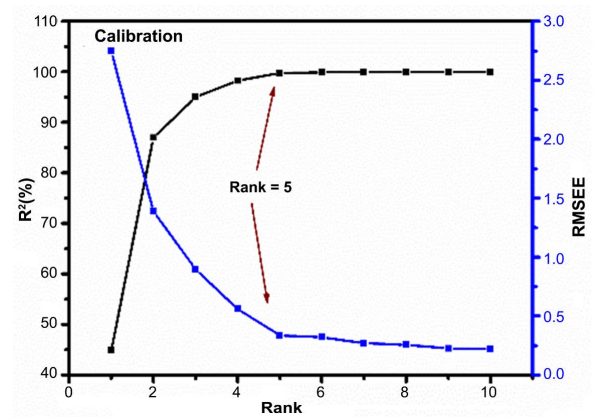

(a)

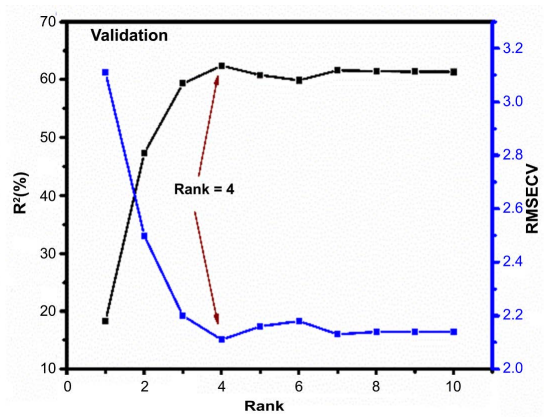

(b)

Figure 7. The correlation coefficient and root mean square of error versus PLS factors for (a) calibration set and (b) validation set.

number (Rank) of 5 and 4 for calibration and validation models, respectively. Obviously, both models are affected by the factors number, moreover, the models improved remarkably after changing the factors to number equal to or more then the optimized one. The differences between the calibration and validation models for default rank number of 1 and for an optimized rank number was illustrated in the Figure 8(a) and Figure 8(b) and a significant improvement in the models was noticed even by looking to the values of $R^{2}, R M S E E$ and $R M S E C V$ for calibration and validation set in Table 2.

\subsubsection{The Influence of Spectral Pre-Processing}

To determine the impact of spectral region selection on the calibration and validation model a comparison between the models parameters $R^{2}$, RMSEC, RMSEV was conducted at full and short range spectral region that contains more spectral data. According to the results presented in Table 3, the more appropriate spectral region for extraction of the best calibration and validation model ranging in 9048.6 - $4026.7 \mathrm{~cm}^{-1}$.

\subsubsection{The Calibration and Validation Results}

In order to set up a robust reliable and an optimized prediction capability, regression models should be determined thought a set of preprocessing methods using optimized PLS factors number and well-established spectral regions. For our investigated PDANS system the best calibration and validation model is the one determined after using offset elimination method, baseline correction and second derivative spectral preprocessing. The optimized rank was 5 , and 4 for calibration and validation model, respectively. The models demonstrate a high correlation between both the lab and predicted values. At $9048.6-4026.7 \mathrm{~cm}^{-1}$, the model parameters $R^{2}, R M S E C$ for calibration model were $99.81 \%$ and 0.196 $\mathrm{nm}$ respectively, for validation model $R^{2}$ and $R M S E C V$ were $70.67 \%, 1.87 \mathrm{~nm}$ respectively. The PLS models constructed for the quantitative evaluation of the particles size estimation were demonstrated to be predictive with high accuracy of our synthesised samples. The optimized models illustrated in Figure 9 was used for estimation of the accurate particle size of known sample ( $\mathrm{dTEM}=135$ 
Table 2. Difference between models parameters determined for default and optimized rank.

\begin{tabular}{ccccc}
\hline & \multicolumn{2}{c}{ Default Rank } & \multicolumn{2}{c}{ Optimized Rank } \\
\hline Model & $R^{2}(\%)$ & $R M S E(\mathrm{~nm})$ & $R^{2}(\%)$ & $R M S E(\mathrm{~nm})$ \\
Calibration & 58.98 & 2.37 & 98.27 & 0.201 \\
Validation & 25.84 & 2.98 & 62.41 & 1.99 \\
\hline
\end{tabular}

Table 3. Comparison between models parameters of full and short range spectral region for an optimized rank.

\begin{tabular}{ccccc}
\hline Spectral region & \multicolumn{2}{c}{ Calibration model } & \multicolumn{2}{c}{ Validation model } \\
\hline Wavenumber $\left(\mathrm{cm}^{-1}\right)$ & $R^{2}$ & $R M S E$ & $R^{2}$ & $R M S E$ \\
$12000.0-4000.0$ & 98.27 & 0.201 & 62.41 & 1.99 \\
$9048.6-4026.7$ & 99.81 & 0.196 & 70.67 & 1.87 \\
$8000.0-4000.0$ & 99.81 & 0.195 & 69.48 & 1.90 \\
$7997.5-5971.1 / 5519.4-4508.9$ & 99.83 & 0.177 & 52.33 & 2.38 \\
$7997.5-5971.1 / 5626.4-3999.7$ & 99.81 & 0.191 & 69.11 & 1.91 \\
\hline
\end{tabular}

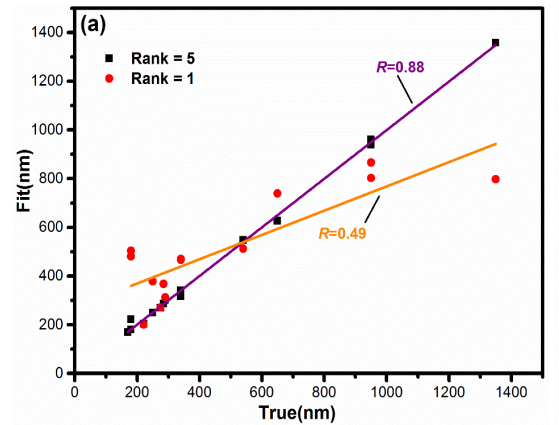

(a)

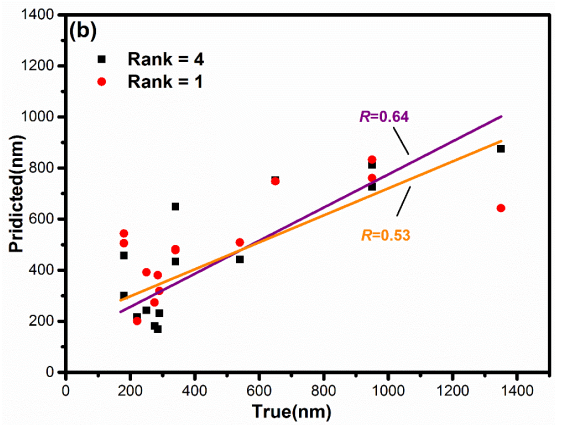

(b)

Figure 8. (a) The calibration and (b) the cross validation models for default and optimized Rank.

$\mathrm{nm}$ ), while the accurate value was estimated to be $135.6 \mathrm{~nm}$ using NIRS model. The analysis of a real data set of PDANS granules size can be given using this robust calibration procedure (see Figure 9) and TEM as reference method.

\section{Conclusion}

In conclusion, our paper introduces NIRS combined with chemometrics as tool for analysis of several physio-chemical properties of designed DDS based on polydopamine nanoparticles at different particles size. The elaborated PDANS as DDS have been monitored with NIRS. Predictive models have been extracted of the NIR versus the TEM granules with and without spectral pre-treatment. Applying robust preprocessing methods as well as model parameters seem to increase the quality of spectral data remarkably compared with non-pre-processed data. Correlation coefficient and root mean square of prediction have been used 


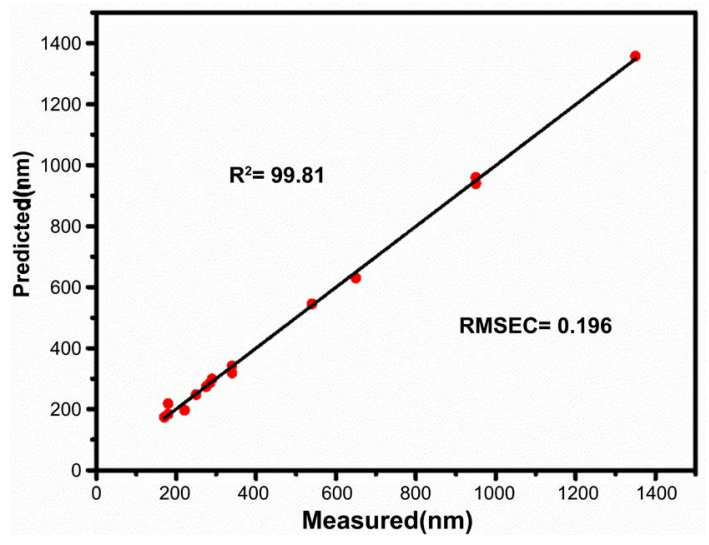

Figure 9. The Correlation curve between NIR predicted and measured values for PDANS particles size.

to select the suitable preprocessing method for our designed system. Our findings allow the PDANS granules which is an illustration of drug nanocarrier to be in-line monitored with NIRS and controlled effectively. As a perspective, a considerable understanding of NIRS combined with chemometrics tool would contribute remarkably for development of high quality DDS. The collected results could be easily extended for analysis of further samples of released DOX form our deigned DDS. A better understanding of the process by NIRS with chemometrics will contribute to developing high-quality therapeutic agent delivery systems.

\section{Acknowledgements}

This work was financially supported by the National Natural Science Foundation of China (Grant No. 51673054), the Harbin City Science and Technology Innovation Talent Foundation (Grant No. 2017RAYXJ003), Shanghai Space Science and Technology Innovation Foundation (Grant No. SAST2017-114).

\section{References}

[1] Shikata, F., Kimura, S., Hattori, Y. and Otsuka, M. (2017) Real-Time Monitoring of Granule Properties during High Shear Wet Granulation by Near-Infrared Spectroscopy with a Chemometrics Approach. RSC Advances, 7, 38307-38317. https://doi.org/10.1039/C7RA05252A

[2] An, X., Li, M., Zheng, L. and Sun, H. (2015) Eliminating the Interference of Soil Moisture and Particle Size on Predicting Soil Total Nitrogen Content Using a NIRs-Based Portable Detector. Computers \& Electronics in Agriculture, 112, 47-53. https://doi.org/10.1016/j.compag.2014.11.003

[3] Cao, W., Mao, C., Chen, W., Lin, H., Krishnan, S. and Cauchon, N. (2006) Differentiation and Quantitative Determination of Surface and Hydrate Water in Lyophilized Mannitol Using NIR Spectroscopy. Journal of Pharmaceutical Sciences, 95, 2077-2086. https://doi.org/10.1002/jps.20706

[4] Shu, J.B., Rani, M., Suryanarayanan, R., Carpenter, J.F. Nayar, R., Manning M.C.J. (2004) Quantification of Glycine Crystallinity by Near-Infrared (NIR) Spectroscopy. Journal of Pharmaceutical Sciences, 93, 2439-2447. 
https://doi.org/10.1002/jps.20153

[5] Rodionov, A., Welp, G., Damerow, L., Berg, T., Amelung, W. and Pätzold, S. (2015) Towards On-the-Go Field Assessment of Soil Organic Carbon Using Vis-Nir Diffuse Reflectance Spectroscopy: Developing and Testing a Novel Tractor-Driven Measuring Chamber. Soil \& Tillage Research, 145, 93-102.

https://doi.org/10.1016/j.still.2014.08.007

[6] Pan, R., Jeffries, J.B., Dreier, T. and Schulz, C. (2016) Measurements of Liquid Film Thickness, Concentration, and Temperature of Aqueous Urea Solution by Nir Absorption Spectroscopy. Applied Physics B, 120, 397-406.

https://doi.org/10.1007/s00340-015-6149-2

[7] Tamburini, E., Vincenzi, F., Costa, S., Mantovi, P., Pedrini, P. and Castaldelli, G. (2017) Effects of Moisture and Particle Size on Quantitative Determination of Total Organic Carbon (TOC) in Soils Using Near-Infrared Spectroscopy. Sensors, 17, 2366. https://doi.org/10.3390/s17102366

[8] Kandpal, L.M., Park, E., Tewari, J. and Cho, B.K. (2015) Spectroscopic Techniques for Nondestructive Quality Inspection of Pharmaceutical Products: A Review. Journal of Biosysetm Engineering, 40, 394-408. https://doi.org/10.5307/JBE.2015.40.4.394

[9] Cabassi, G., Profaizer, M., Marinoni, L., Rizzi, N. and Cattaneo, T. (2013) Estimation of Fat Globule Size Distribution in Milk Using an Inverse Light Scattering Model in the Near Infrared Region. Journal of Near Infrared Spectroscopy, 21, 359-373. https://doi.org/10.1255/jnirs.1070

[10] Rahoui, N., Jiang, B., Taloub, N. and Huang, Y.D. (2017) Spatio-Temporal Control Strategy of Drug Delivery Systems Based Nano Structures. Journal of Controlled Release, 255, 176. https://doi.org/10.1016/j.jconrel.2017.04.003

[11] Song, J., Xie, J., Li, C., Lu, J.H., Meng, Q.F., Yang, Z., Lee, R.J., Wang, D. and Teng, L.S. (2014) Near Infrared Spectroscopic (NIRS) Analysis of Drug-Loading Rate and Particle Size of Risperidone Microspheres by Improved Chemometric Model. International Journal of Pharmaceutics, 472, 296-303. https://doi.org/10.1016/j.ijpharm.2014.06.033

[12] Rahoui, N., Jiang, B., Pan, H.T. and Huang, Y.D. (2016) Spectroscopy Strategy for Solid Propellants Quality Control. Applied Spectroscopy Reviews, 51, 431-450. https://doi.org/10.1080/05704928.2016.1145124

[13] Hagiwara, K., Kurihara, K., Honma, M., Yamamoto, J. and Shinohara, F. (2018) PEG-Modification on the Endo-Position of an Antisense Oligonucleotide Increases Tumor Accumulation via the EPR Effect. Journal of Biomaterials Science, Polymer Edition, 4, 448-459. https://doi.org/10.1080/09205063.2017.1422853

[14] Lacerda, M.P.C., Demattê, J.A.M., Sato, M.V., Fongaro, C.T., Gallo, B.C. and Souza, A.B. (2016) Tropical Texture Determination by Proximal Sensing Using a Regional Spectral Library and Its Relationship with Soil Classification. Remote Sensing, 8, 1-20. https://doi.org/10.3390/rs8090701

[15] Lai, G., Zhang, H., Yong, J. and Yu, A. (2013) In Situ Deposition of Gold Nanoparticles on Polydopamine Functionalized Silica Nanosphere for Ultrasensitive Nonenzymatic Electrochemical Immunoassay. Biosensors \& Bioelectronics, 47, 178-183. https://doi.org/10.1016/j.bios.2013.03.029

[16] Ma, L., Jing, Z., Nan, L., Ren, X. and Liu (2014) In Situ Green Synthesis of au Nanoparticles onto Polydopamine-Functionalized Graphene for Catalytic Reduction of Nitrophenol. RSC Advances, 4, 64816-64824. https://doi.org/10.1039/C4RA11950A 
[17] Falade, T.D.O., Sultanbawa, Y., Fletcher, M.T. and Fox, G. (2017) Near Infrared Spectrometry for Rapid Non-Invasive Modelling of Aspergillus-Contaminated Maturing Kernels of Maize (Zea mays L.). Agriculture, 7, 77. https://doi.org/10.3390/agriculture7090077

[18] Yu, H., Ying, Y., Fu, X. and Lu, H. (2006) Quality Determination of Chinese Rice Wine Based on Fourier Transform near Infrared Spectroscopy. Journal of Near Infrared Spectroscopy, 14, 37-44. https://doi.org/10.1255/jnirs.584

[19] Miller, N.J. and Miller, J.C. (2010) Statistics and Chemometrics for Analytical Chemistry. 6th Edition, Pearson Education Limited, Gosport, 241-244.

[20] Agelet, L.E. (2011) Single Seed Discriminative Applications Using near Infrared Technologies. PhD Thesis, Iowa State University, Ames. 